\title{
APPROXIMATION OF SOLUTIONS TO EVOLUTION INTEGRODIFFERENTIAL EQUATIONS
}

\author{
D. BAHUGUNA \\ IIT, Department of Mathematics \\ Madras 600 036, INDIA \\ S.K. SRIVASTAVA \\ Lucknow University \\ Department of Mathematics and Astronomy \\ Lucknow 226 007, INDIA
}

(Received May, 1995; Revised October, 1995)

\begin{abstract}
In this paper we study a class of evolution integrodifferential equations. We first prove the existence and uniqueness of solutions and then establish the convergence of Galerkin approximations to the solution.
\end{abstract}

Key words: Evolution Equation, Fixed Point Method, Galerkin Approximation.

AMS (MOS) subject classifications: $34 \mathrm{G} 20,35 \mathrm{~A} 35,35 \mathrm{~K} 55$.

\section{Introduction}

Let $\mathbf{H}$ be a separable real Hilbert space. We consider the following integrodifferential equation in $\mathbf{H}$ :

$$
\begin{aligned}
& \frac{d u(t)}{d t}=-A u(t)+M(u(t))+\int_{0}^{t} g(t-s) k(u(s)) d s, \quad t>0, \\
& u(0)=\phi,
\end{aligned}
$$

where $A$ is a closed, positive definite, selfadjoint linear operator with dense domain $D(A)$ in $\mathbf{H}$. We assume that $A$ has a pure point spectrum $0<\lambda_{0} \leq \lambda_{1} \leq \ldots$ and a corresponding complete orthonormal system $\left\{u_{i}\right\}$ so that $A u_{i}=\lambda_{i} u_{i}$ and $\left(u_{i}, u_{j}\right)=\delta_{i j},(\cdot, \cdot)$ is the inner product in $\mathbf{H}$ and $\delta_{i j}$ is the Kronecker delta function. These assumptions on $A$ guarantee that $-A$ generates an analytic semigroup $e^{-t A}$. The nonlinear operators $M$ and $k$ are defined on $D\left(A^{\alpha}\right)$ for some $\alpha$, $0<\alpha<1$ and $\phi$ is in $D(A)$. The map $g$ is a real-valued continuous function defined on $\mathbf{R}_{+}$.

The existence and uniqueness of solutions to (1) is closely associated with the existence and uniqueness of solutions to the integrodifferential equation

$$
u(t)=e^{-t A} \phi+\int_{0}^{t} e^{-(t-s) A}[M(u(s))+K(u)(s)] d s,
$$


where the nonlinear Volterra operator $K(u)(t)=\int_{0}^{t} g(t-s) k(u(s)) d s$.

The present work is closely related to the paper of Miletta [5] in which he has investigated the abstract evolution equation

$$
\begin{gathered}
\frac{d u(t)}{d t}=A u(t)+M(u(t)), \\
u(0)=\phi,
\end{gathered}
$$

and the related integral equation

$$
u(t)=e^{-t A} \phi+\int_{0}^{t} e^{-(t-s) A} M(u(s)) d s .
$$

For initial studies related to $(3,4)$ we refer to Segal [9] and Murakami [6]. For a Lipschitz continuous $M$ and $\phi$ in $D\left(A^{\alpha}\right)$ (4) has a strong solution on some maximal interval of existence, and if the interval of existence is bounded, then there are some blow-up results associated with the solution. We also refer to the papers of Bazley $[1,2]$.

We first establish the existence and uniqueness results for the integral equation (2). The method used is similar to the one used by Miletta [5]. The solution to (2) is obtained as the limit of the solutions to the integral equations satisfied by the Galerkin approximations. As remarked by Miletta [5], the assumptions on the nonlinear maps are not general as far as the existence results are concerned, but these assumptions give uniform convergence of the approximations.

We assume the following condition on the nonlinear maps $M$ and $k$ :

(L) The nonlinear maps $M$ and $k$, defined on $D\left(A^{\alpha}\right)$ into $\mathbf{H}$ for some $\alpha, 0<\alpha<1$, are continuous and for each $r>0$ there exist positive constants $C_{M}(r)$ and $C_{k}(r)$ such that

(a) $\quad\|M(u)\| M \leq C_{M}(r)$ and $\|k(u)\| \leq C_{k}(r)$ for $u \in D\left(A^{\alpha}\right)$ with $\left\|A^{\alpha} u\right\| \leq r$,

(b) $\quad\left\|M\left(u_{1}\right)-M\left(u_{2}\right)\right\| \leq C_{M}(r)\left\|A^{\alpha}\left(u_{1}-u_{2}\right)\right\|$ and

$$
\left\|k\left(u_{1}\right)-k\left(u_{2}\right)\right\| \leq C_{k}(r)\left\|A^{\alpha}\left(u_{1}-u_{2}\right)\right\|
$$

for $u_{i} \in D\left(A^{\alpha}\right)$ with $\left\|A^{\alpha} u_{i}\right\| \leq r$ for $i=1,2$.

For existence, uniqueness and regularity results we may consider more general nonautonomous nonlinearities $M(t, u)$ and $k(t, u)$ in (1) which satisfy the following conditions (cf. Bahuguna $[3])$ :

$$
\begin{gathered}
\left\|M\left(t_{1}, u_{1}\right)-M\left(t_{2}, u_{2}\right)\right\| \leq C_{M}(r)\left[\left|t_{1}-t_{2}\right|^{\beta}+\left\|A^{\alpha}\left(u_{1}-u_{2}\right)\right\|\right] \\
\left\|k\left(t_{1}, u_{1}\right)-k\left(t_{2}, u_{2}\right)\right\| \leq C_{k}(r)\left[\left|t_{1}-t_{2}\right|^{\mu}+\left\|A^{\alpha}\left(u_{1}-u_{2}\right)\right\|\right]
\end{gathered}
$$

for $u_{i} \in D\left(A^{\alpha}\right)$ with $\left\|u_{i}\right\| \leq r, i=1,2$, and for some $\beta, \mu$ with $0<\beta, \mu<1$.

\section{Existence and Uniqueness}

This section is devoted to establishing the existence and uniqueness of the solutions to the integral equation $(2)$ on $[0, T]$ for some $0<T<\infty$. Under the assumptions mentioned in $\S 1$, we prove the following existence and uniqueness result. 
Theorem 2.1: Suppose the assumption (L) holds and $\phi \in D(A)$. Then there exist a $T$, $0<T<\infty$ and a unique in $\mathrm{e}\left([0, T], D\left(A^{\alpha}\right)\right)$ satisfying $(2)$ on $[0, T]$.

We shall prove Theorem 2.1 with the help of several lemmas to be proved in this section. Let $T_{0}, 0<T_{0}<\infty$ be fixed, but arbitrary.

Let

$$
T=\min \left\{T_{0},\left[\frac{1}{2}(1-\alpha) F^{-1} C(R)^{-1}\right]^{\frac{1}{(1-\alpha)}}\right\},
$$

where $F$ is such that $\left\|A^{\alpha} e^{-t A}\right\| \leq F t^{-\alpha}, R=F\|\phi\|_{\alpha}+1$ and

$$
C(r)=C_{M}(r)+T_{0} g_{0} C_{k}(r)
$$

for $r>0$ and

$$
g_{0}=\max _{0 \leq t \leq T_{0}}|g(t)|
$$

We shall denote by $X^{\alpha}$ the Banach space $\mathrm{C}\left([0, T], D\left(A^{\alpha}\right)\right)$ endowed with the norm

$$
\|u\|_{\alpha} \sup _{0 \leq t \leq T}\left\|A^{\alpha} u(t)\right\| .
$$

Let $\mathbf{H}_{n}$ denote the subspace of $\mathbf{H}$ generated by $\left\{u_{0}, u_{1}, \ldots, u_{n}\right\}$ and $P^{n}: \mathbf{H} \rightarrow \mathbf{H}_{n}$ the associated projections. For each $n$, we define

$$
\begin{gathered}
M_{n}(u)=M\left(P^{n} u\right) \\
k_{n}(u)=k\left(P^{n} u\right), \\
K_{n}(u)(t)=\int_{0}^{t} g(t-s) k_{n}(u(s)) d s .
\end{gathered}
$$

For $n=1,2, \ldots$ we define a map $S_{n}$ on $B_{R}\left(X^{\alpha}\right)=\left\{u \in X^{\alpha} \mid\|u\|_{\alpha} \leq R\right\}$ as

$$
S_{n} u=e^{-t A} \phi+\int_{0}^{t} e^{-(t-s) A}\left[M_{n}(u(s))+K_{n}(u)(s)\right] d s .
$$

Lemma 2.1: The map

$$
S_{n}: B_{R}\left(X^{\alpha}\right) \rightarrow B_{R}\left(X^{\alpha}\right)
$$

is a contraction.

Proof: We note that for $u \in B_{R}\left(X^{\alpha}\right)$,

$$
\begin{gathered}
\left\|S_{n} u\right\| \leq \sup _{0 \leq t \leq T}\left\|e^{-t A} A^{\alpha} \phi\right\|+\sup _{0 \leq t \leq T} \int_{0}^{t}\left\|A^{\alpha} e^{-(t-s) A}\right\|\left\|M_{n}(u(s))+K_{n}(u)(s)\right\| d s \\
\leq F\|\phi\|_{\alpha}+\frac{F C(R) T^{1-\alpha}}{1-\alpha} \leq R .
\end{gathered}
$$

Thus $S_{n}: B_{R}\left(X^{\alpha}\right) \rightarrow B_{R}\left(X^{\alpha}\right)$. Now, for $u_{1}$ and $u_{2}$ in $B_{R}\left(X^{\alpha}\right)$, we have 


$$
\begin{gathered}
\left\|M_{n}\left(u_{1}(s)\right)-M_{n}\left(u_{2}(s)\right)\right\| \leq C_{M}(R)\left\|u_{1}-u_{2}\right\|_{\alpha} \\
\left\|K_{n}\left(u_{1}\right)(s)-K_{n}\left(u_{2}\right)(s)\right\| \leq g_{0} \int_{0}^{t}\left\|k_{n}\left(u_{1}(\tau)\right)-k_{n}\left(u_{2}(\tau)\right)\right\| d \tau \\
\leq g_{0} T_{0} C_{k}(R)\left\|u_{1}-u_{2}\right\|_{\alpha} .
\end{gathered}
$$

Hence for $u_{1}$ and $u_{2}$ in $B_{R}\left(X^{\alpha}\right)$, we have

$$
\begin{gathered}
\left\|S_{n}\left(u_{1}\right)-S_{n}\left(u_{2}\right)\right\|_{\alpha \leq} \sup _{0 \leq t \leq T} \int_{0}^{t}\left\|A^{\alpha} e^{-(t-s) A}\right\|\left[\left\|M_{n}\left(u_{1}(s)\right)-M_{n}\left(u_{2}(s)\right)\right\|\right. \\
\left.+\left\|K_{n}\left(u_{1}\right)(s)-K_{n}\left(u_{2}\right)(s)\right\|\right] d s \\
\leq \frac{F C(R) T^{1-\alpha}}{1-\alpha}\left\|u_{1}-u_{2}\right\|_{\alpha} \\
\leq \frac{1}{2}\left\|u_{1}-u_{2}\right\|_{\alpha} .
\end{gathered}
$$

Thus, $S_{n}$ is a contraction on $B_{R}\left(X^{\alpha}\right)$ and therefore there exists a unique $u_{n}$ in $B_{R}\left(X^{\alpha}\right)$ such that

$$
u_{n}(t)=e^{-t A} \phi+\int_{0}^{t} e^{-(t-s) A}\left[M_{n}(u(s))+K_{n}(u)(s)\right] d s .
$$

We shall assume throughout that $\phi$ is in $D(A)$ unless otherwise stated.

Lemma 2.2: We have

$$
u_{n}:[0, T] \rightarrow D\left(A^{\beta}\right)
$$

for all $0 \leq t \in[0, T]$ and $0 \leq \beta<1$.

Corollary 2.1: There exists a constant $U_{0}$ independent of $n$ such that

$$
\left\|A^{\beta} u(t)\right\| \leq U_{0}
$$

for all $t \in[0, T]$ and $0 \leq \beta<1$.

For proofs of Lemma 2.2 and Corollary 2.1, we refer to the proofs of Lemma 1 and Corollaries 1 and 2 in [5].

Lemma 2.3:

$$
\sup _{\{n \geq m, 0 \leq t \leq T\}}\left\|A^{\alpha}\left[u_{n}(t)-u_{m}(t)\right]\right\| \rightarrow 0 \text { as } m \rightarrow \infty .
$$

Proof: For $n \geq m$, we have

$$
\begin{gathered}
\left\|M_{n}\left(u_{n}(s)\right)-M_{m}\left(u_{m}(s)\right)\right\| \leq\left\|M_{n}\left(u_{n}(s)\right)-M_{n}\left(u_{m}(s)\right)\right\|+\left\|M_{n}\left(u_{m}(s)\right)+M_{m}\left(u_{m}(s)\right)\right\| \\
\leq C_{M}(R)\left\|A^{\alpha}\left[u_{n}(s)-u_{m}(s)\right]\right\|+C_{M}(r)\left\|A^{\alpha-\beta}\left(P^{n}-P^{m}\right) A^{\beta} u_{m}(s)\right\| \\
\leq C_{M}(R)\left\|A^{\alpha}\left[u_{n}(s)-u_{m}(s)\right]\right\|+\frac{C_{M}(R)}{\lambda_{m}^{\beta-\alpha}}\left\|A^{\beta} u_{m}(s)\right\|
\end{gathered}
$$


Similarly,

$$
\left\|k_{n}\left(u_{n}(s)\right)-k_{m}\left(u_{m}(s)\right)\right\| \leq C_{k}(R)\left\|A^{\alpha}\left[u_{n}(s)-u_{m}(s)\right]\right\|+\frac{C_{k}(R)}{\lambda_{m}^{\beta-\alpha}}\left\|A^{\beta} u_{m}(s)\right\| .
$$

From (14) and Corollary 2.1, for $n \geq m$, we have

$$
\begin{gathered}
\left\|K_{n}\left(u_{n}\right)(s)-K_{m}\left(u_{m}\right)(s)\right\| \leq g_{0} \int_{0}^{s}\left\|K_{n}\left(u_{n}(\tau)\right)-K_{m}\left(u_{m}(\tau)\right)\right\| d \tau \\
\leq \frac{T_{0} g_{0} C_{k}(R) U_{0}}{\lambda_{m}^{\beta-\alpha}}+T_{0} g_{0} C_{k}(R) \sup _{0 \leq \tau \leq s}\left\|A^{\alpha}\left[u_{n}(\tau)-u_{m}(\tau)\right]\right\| .
\end{gathered}
$$

Also, from (14) we have

$$
\left\|M_{n}\left(u_{n}(s)\right)-M_{m}\left(u_{m}(s)\right)\right\| \leq \frac{C_{M}(R) U_{0}}{\lambda_{m}^{\beta-\alpha}}+C_{M}(R) \sup _{0 \leq \tau \leq s}\left\|A^{\alpha}\left[u_{n}(\tau)-u_{m}(\tau)\right]\right\| .
$$

Using the estimates of (16) and (17) in the integral equation (13) we obtain that

$$
\begin{aligned}
\left\|A^{\alpha}\left[u_{n}(t)-u_{m}(t)\right]\right\| \leq & \int_{0}^{t}\left\|A^{\alpha} e^{-(t-s) A}\right\|\left[\| M_{n}\left(u_{n}\left(u_{n}(s)\right)-M_{m}\left(u_{m}(s)\right) \|\right.\right. \\
& \left.+\left\|K_{n}\left(u_{n}\right)(s)-K_{m}\left(u_{m}\right)(s)\right\|\right] d s \\
\leq & \frac{C(R) F U_{0} T^{1-\alpha}}{\lambda_{m}^{\beta-\alpha}(1-\alpha)}+C(R) F \int_{0}^{t}(t-s)^{-\alpha} \sup _{0 \leq \tau \leq s}\left\|A^{\alpha}\left[u_{n}(\tau)-u_{m}(\tau)\right]\right\| d s
\end{aligned}
$$

The above inequality implies that

$$
\begin{gathered}
\sup _{0 \leq \tau \leq t}\left\|A^{\alpha}\left[u_{n}(\tau)-u_{m}(\tau)\right]\right\| \\
\leq \frac{C(R) F U_{0} T^{1-\alpha}}{\lambda_{m}^{\beta-\alpha}(1-\alpha)}+C(R) F \int_{0}^{t}(t-s) \sup _{0 \leq \tau \leq s}^{-\alpha}\left\|A^{\alpha}\left[u_{n}(\tau)-u_{m}(\tau)\right]\right\| d s .
\end{gathered}
$$

Applying the Gronwall's inequality in (18) we get

$$
\sup _{0 \leq \tau \leq T}\left\|A^{\alpha}\left[u_{n}(\tau)-u_{m}(\tau)\right]\right\| \leq \frac{C(R) F U_{0} T^{1-\alpha}}{\lambda_{m}^{\beta-\alpha}(1-\alpha)}\left[1+\frac{C(R) F T^{1-\alpha}}{1-\alpha} e^{\left.\frac{C(R) F T^{1-\alpha}}{1-\alpha}\right] .}\right.
$$

Taking the limit as $m \rightarrow \infty$ on both the sides, we get the required result.

Proof of Theorem 2.1: From Lemma 2.3, it follows that there exists a unique $u \in B_{R}\left(X^{\alpha}\right)$ such that

$$
\sup _{0 \leq \tau \leq T}\left\|A^{\alpha}\left[u_{n}(\tau)-u(\tau)\right]\right\| \rightarrow 0 \text { as } n \rightarrow \infty
$$

The continuity of $M$ and $k$ imply that

$$
\left\|M_{n}\left(u_{n}(t)\right)-M(u(t))\right\|+\left\|k_{n}\left(u_{n}\right)(t)-k(u)(t)\right\| \rightarrow 0 \text { as } n \rightarrow \infty .
$$

We may take the limit as $n \rightarrow \infty$ in (13) to assert that $u$ satisfies the integral equation

$$
u(t)=e^{-t A} \phi+\int_{0}^{t} e^{-(t-s) A}[M(u(s))+K(u)(s)] d s .
$$


Now we prove the uniqueness. Suppose that $u_{i} \in X^{\alpha}, i=1,2$; are two solutions to (20) on some interval $[0, T]$. Then for $R>0$ large enough, we have

$$
\sup _{0 \leq \tau \leq t}\left\|A^{\alpha}\left[u_{1}(\tau)-u_{2}(\tau)\right]\right\| \leq C(R) F \int_{0}^{t}(t-s)^{-\alpha} \sup _{0 \leq \tau \leq s}\left\|A^{\alpha}\left[u_{1}(\tau)-u_{2}(\tau)\right]\right\| d s .
$$

The uniqueness follows from the application of Gronwall's inequality in (21) and the fact that for any $u \in X^{\alpha}$,

$$
\|u\| \leq \lambda_{0}^{-\alpha}\|u\|_{\alpha}
$$

\section{Faedo-Galerkin Approximations}

The solution $u \in X^{\alpha}$ of the integral equation (20) and $u_{n} \in X^{\alpha}$ satisfying the approximate integral equation (13) have the representations

$$
\begin{gathered}
u(t)=\sum_{i=0}^{\infty} \alpha_{i}(t) u_{i}, \quad \alpha_{i}(t)=\left(u(t), u_{i}\right) \quad i=0,1, \ldots \\
u_{n}(t)=\sum_{i=0}^{n} \alpha_{i}^{n}(t) u, \quad \alpha_{i}^{n}(t)=\left(u_{n}(t), u_{i}\right) \quad i=0,1, \ldots .
\end{gathered}
$$

Projecting the integral (20) on $\mathbf{H}_{n}$, we get the Faedo-Galerkin approximation

Setting

$$
P^{n} v(t)=e^{-t A} P^{n} \phi+\int_{0}^{t} e^{-(t-s) A} P^{n}\left[M\left(P^{n} v(s)\right)+\int_{0}^{s} g(s-\tau) k\left(P^{n} v(\tau)\right) d \tau\right] d s .
$$

$$
v=v_{n}(t)=\sum_{i=0}^{n} \alpha_{i}^{n}(t) u_{i}
$$

we obtain a system of first order integrodifferential equations

$$
\frac{d \alpha_{i}^{n}(t)}{d t}+\lambda_{i} \alpha_{i}^{n}(t)=M_{i}^{n}\left(\alpha_{0}^{n}(t), \ldots, \alpha_{n}(t)\right)+\int_{0}^{t} g(t-s) k_{i}^{n}\left(\alpha_{0}^{n}(s), \ldots, \alpha_{n}^{n}(s)\right) d s,
$$

where

$$
\begin{aligned}
M_{i}^{n}\left(\alpha_{0}^{n}(t), \ldots, \alpha_{n}^{n}(t)\right) & =\left(M\left(\sum_{i=0}^{n} \alpha_{i}^{n}(t) u_{i}\right), u_{i}\right), \\
k_{i}^{n}\left(\alpha_{0}^{n}(t), \ldots, \alpha_{n}^{n}(t)\right) & =\left(k\left(\sum_{i=0}^{n} \alpha_{i}^{n}(t) u_{i}\right), u_{i}\right) .
\end{aligned}
$$

We observe that $v_{n}(t)=P^{n} u_{n}(t)$. We have the following convergence theorem.

Theorem 3.1:

$$
\lim _{n \rightarrow \infty} \sup _{0 \leq t \leq T}\left(\sum_{i=0}^{n} \lambda_{i}^{2 \alpha}\left[\alpha_{i}(t)-\alpha_{i}^{n}(t)\right]^{2}\right)=0
$$


Proof:

Thus

$$
\begin{gathered}
A\left[u(t)-u_{n}(t)\right]=A\left[\sum_{i=0}^{\infty}\left(\alpha_{i}(t)-\alpha_{i}^{n}(t)\right) u_{i}\right] \\
=\sum_{i=0}^{\infty} \lambda_{i}^{\alpha}\left(\alpha_{i}(t)-\alpha_{i}^{n}(t)\right) u_{i}
\end{gathered}
$$

$$
\left\|A^{\alpha}\left[u(t)-u_{n}(t)\right]\right\|^{2} \geq \sum_{i=0}^{n} \lambda_{i}^{2 \alpha}\left[\alpha_{i}(t)-\alpha_{i}^{n}(t)\right]^{2} .
$$

The required result follows from Lemma 2.3.

\section{Applications}

We consider the following integrodifferential equation

$$
\begin{gathered}
u_{t}-\Delta u=f_{1}(u, \nabla u)+\int_{0}^{t} g(t-s) f_{2}(u(s), \nabla u(s)) d s, \quad x \in \Omega, t>0, \\
u(0, x)=u_{0}(x),
\end{gathered}
$$

where $\Omega \subseteq \mathbf{R}^{3}$ is a bounded domain with sufficiently smooth boundary $\partial \Omega, \Delta$ is the 3-dimensional Laplacian, $g: \mathbf{R}_{+} \rightarrow \mathbf{R}$ is a continuous function and $f_{i}(u, p),(u, p) \in \mathbf{R} \times \mathbf{R}^{3}, i=1,2$, are locally Lipschitz continuous functions of all its arguments and there is a continuous function $\rho: \mathbf{R}_{+} \rightarrow \mathbf{R}_{+}$ and a real constant $\gamma, 1 \leq \gamma<3$ such that for $i=1,2$, we have

$$
\begin{gathered}
\left|f_{i}(u, p)\right| \leq \rho(|u|)\left(1+|p|^{\gamma}\right) \\
\left|f_{i}(u, p)-f_{i}(u, q)\right| \leq \rho(|u|)\left(1+|p|^{\gamma-1}+|q|^{\gamma-1}\right), \\
\left|f_{i}(u, p)-f_{i}(v, p)\right| \leq \rho(|u|+|v|)\left(1+|p|^{\gamma}\right)|u-v| .
\end{gathered}
$$

We refer to Pazy [7] for the case $f_{2} \equiv 0$ in (27). See also Fujita and Kato [4] and Ponce [8] for related problems. For more general problems, we refer to Simon [10] and references therein.

We reformulate (27) as an abstract integrodifferential equation (1) in the real Hilbert space $\mathbf{H}=L^{2}(\Omega)$ where $A=-\Delta+c I$, for some $c>0$ with $D(A)=\mathbf{H}^{2}(\Omega) \cap \mathbf{H}_{0}^{1}(\Omega), \mathbf{H}^{2}(\Omega)$ and $\mathbf{H}_{0}^{1}(\Omega)$ are Sobolev spaces (cf. $§ 7.1$ in Pazy [7]), $I$ is the identity operator and

$$
\begin{gathered}
M(u)=f_{1}(u, \nabla u)+c u, \\
k(u)=f(u, \nabla u), \\
\phi(x)=u_{0}(x) .
\end{gathered}
$$

We observe that for $\alpha, \max \left\{\frac{3}{4}, \frac{5 \gamma-3}{4 \gamma}\right\}<\alpha<1$, all the assumptions of Theorem 2.1 are satisfied (cf. Corollary 2.3.7 on page 51 and the estimate (4.20) on page 245 in Pazy [7]). Thus, we may obtain the corresponding existence, uniqueness and convergence results for (27). 


\section{Acknowledgements}

The authors wish to thank the referee for his/her valuable remarks.

\section{References}

[1] Bazley, N., Approximation of wave equations with reproducing nonlinearities, Nonlinear Analysis: TMA 3 (1979), 539-546.

[2] Bazley, N., Global convergence of Fadeo-Galerkin approximations to nonlinear wave equations, Nonlinear Analysis: TMA 4 (1980), 503-507.

[3] Bahuguna, D., Strongly damped semilinear equations, J. Appl. Math. Stoch. Anal., 8:4 (1995), 397-404.

[4] Fujita, H. and Kato, T., On the Navier-Stokes initial value problem I, Arch. Rat. Mech. Anal. 16 (1964), 269-315.

[5] Miletta, P.D., Approximation of solutions to evolution equations, Math. Methods in the Appl. Sci. 17 (1994), 753-763.

[6] Murakami, H., On linear ordinary and evolution equations, Funkcial Ekvac. 9 (1966), 151162.

[7] Pazy, A., Semigroups of Linear Operators and Applications to Partial Differential Equations, Springer-Verlag, New York 1983.

[8] Ponce, G., Global existence of small solutions to a class of nonlinear evolution equations, Nonlinear Analysis: TMA 9 (1985), 399-418.

[9] Segal, I., Nonlinear semigroups, Ann. Math. 78 (1963), 339-346.

[10] Simon, L, Strongly nonlinear parabolic functional differential equations, Annales Univ. Sci. Budapest 37 (1994), 215-228. 


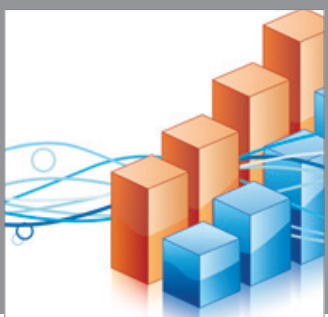

Advances in

Operations Research

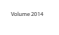

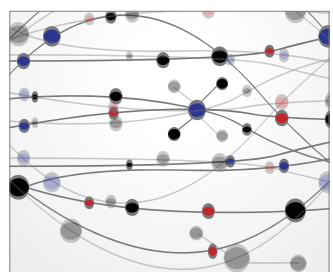

\section{The Scientific} World Journal
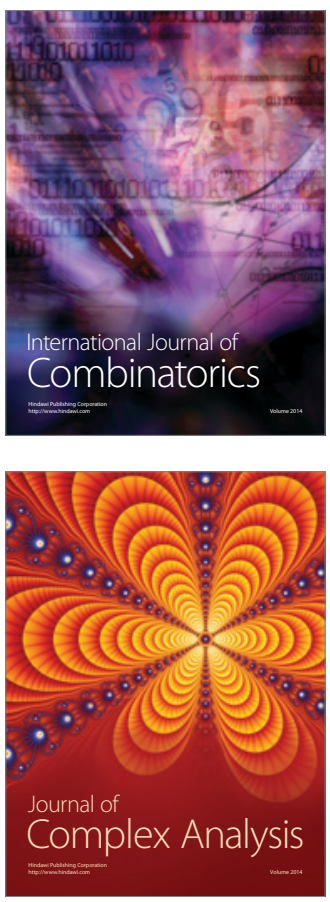

International Journal of

Mathematics and

Mathematical

Sciences
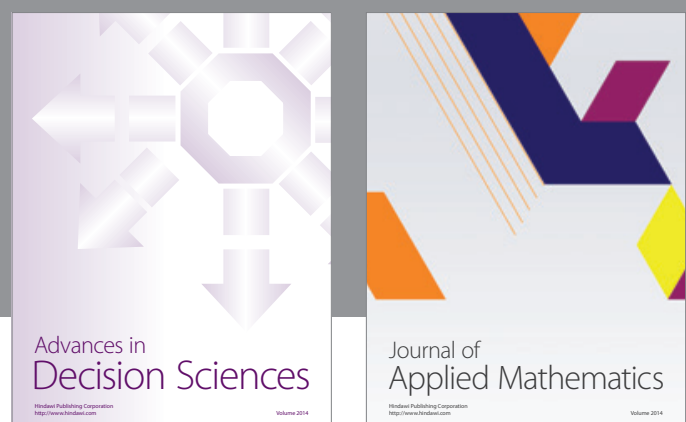

Journal of

Applied Mathematics
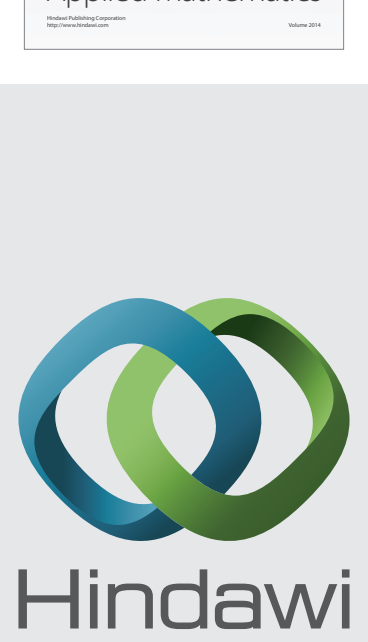

Submit your manuscripts at http://www.hindawi.com
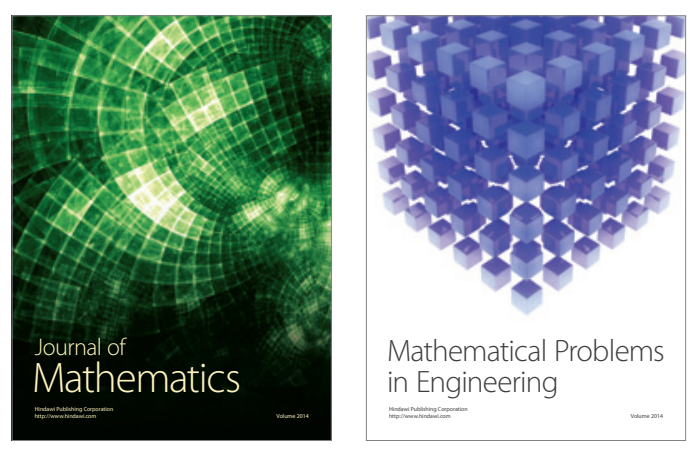

Mathematical Problems in Engineering
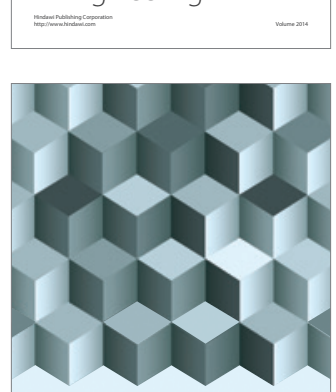

Journal of

Function Spaces
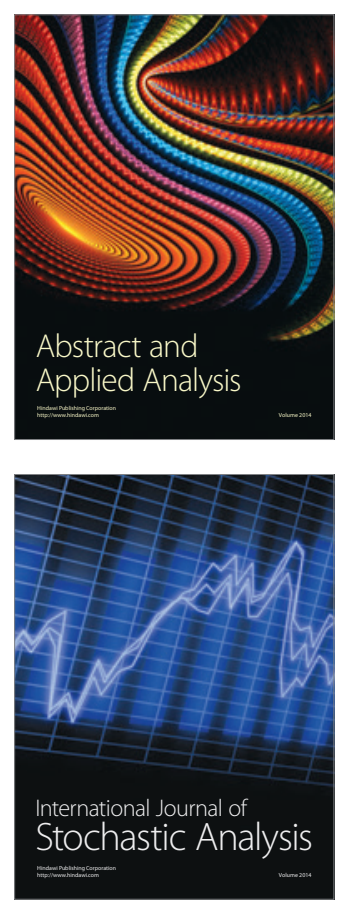

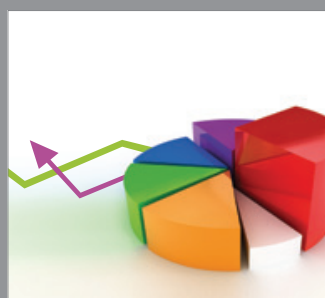

ournal of

Probability and Statistics

Promensencen
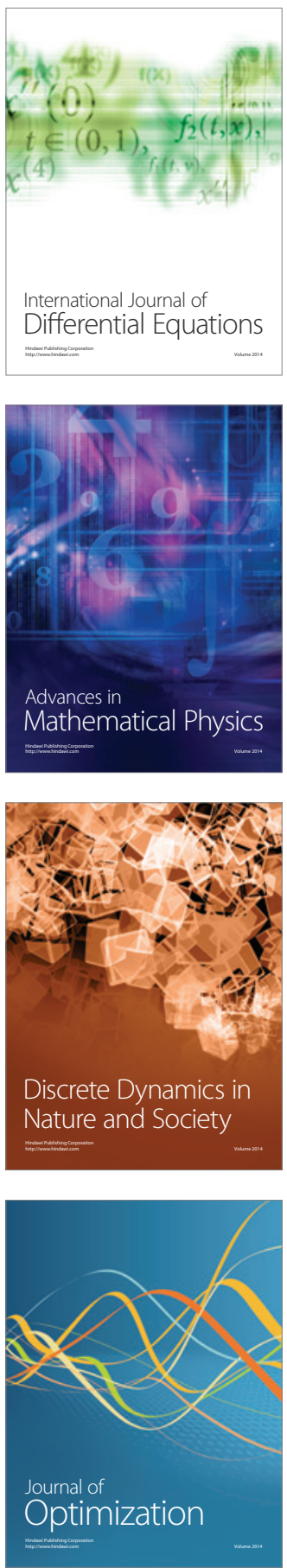